\title{
Disparités d'espérance de vie selon le revenu dans la ville de Toronto et dans la région de Peel, en Ontario
}

\section{J. Stratton, M. Sc. S. (1); D. L. Mowat, FRCPC (1,2,3,4); R. Wilkins, M. Urb. (5,6); M. Tjepkema, M.S.P. (5)}

Cet article a fait l'objet d'une évaluation par les pairs.

\section{Résumé}

Introduction : Pour comprendre le faible gradient de mortalité selon le revenu du quartier observé dans une étude antérieure, nous avons utilisé les données individuelles de l'Étude canadienne de suivi de la mortalité selon le recensement de 1991 à 2001 afin d'examiner les disparités d'espérance de vie et de probabilité de survie jusqu'à l'âge de 75 ans selon le revenu, dans la ville de Toronto et la région de Peel.

Méthodologie : Nous avons calculé des tables de survie selon le sexe et le quintile de suffisance du revenu pour tous les membres de la cohorte ainsi que pour les immigrants et les non-immigrants pris séparément.

Résultats : Pour tous les membres de la cohorte, tant chez les hommes que chez les femmes et tant chez les immigrants que chez les non-immigrants, on a observé un gradient manifeste, l'espérance de vie augmentant graduellement avec le quintile de revenu. Toutefois, les disparités selon le revenu étaient beaucoup plus élevées lorsque l'analyse se limitait aux non-immigrants. Le gradient plus faible observé chez les immigrants semblait refléter la plus grande proportion de nouveaux immigrants dans les quintiles de revenu inférieurs.

Conclusion : Ces observations font ressortir l'importance de recourir, dans la mesure du possible, aux données sur le revenu personnel et d'inclure le statut d'immigrant et la période d'immigration dans l'évaluation des indicateurs de la santé, en particulier dans les régions comptant une forte proportion d'immigrants.

Mots-clés : étude de cohorte, mortalité, probabilité de survie, effet de l'immigrant en bonne santé, Canada

\section{Introduction}

Le " gradient de santé selon le revenu ", c'est-à-dire un meilleur état de santé allant de pair avec un revenu plus élevé, a été démontré à plusieurs reprises, tant au Canada qu'aux États-Unis, par des études portant sur divers indicateurs de la santé dont la mortalité ${ }^{1,2}$, l'espérance de $v^{2}{ }^{2}$, la qualité de vie liée à la santé $e^{3}$ et l'incapacité ${ }^{3,4}$. Les personnes moins favorisées sur le plan socioéconomique présentent un moins bon état de santé que les personnes plus aisées, comme l'indique toute une gamme de mesures. Le gradient socioéconomique n'est pas statique : il varie dans le temps selon l'âge et le sexe, mais aussi selon la mesure de la santé et la sous-population étudiée.
Dans le cadre d'une analyse récente, à petite échelle, portant sur la relation entre l'espérance de vie et le revenu du quartier dans la région de Peel (située juste à l'ouest de la ville de Toronto), nous avons utilisé la Base de données sur la mortalité de l'Ontario pour l'année 2005 et les données du recensement de 2006 concernant le pourcentage de la population à faible revenu par secteur de recensement (annexe, tableau A). Nos résultats ont indiqué que l'espérance de vie était semblable dans tous les quintiles de revenu du quartier, et ce, tant chez les hommes que chez les femmes. Manifestement, le gradient attendu n'était pas présent. Nous avons donc effectué une analyse des données de cohorte mortalité-recensement déjà couplées $^{5}$ afin de nous aider à comprendre le comment et le pourquoi de cette situation. Nous avons formulé l'hypothèse que le pourcentage élevé d'immigrants (49 \%) dans cette région pourrait avoir occulté le gradient de mortalité selon le revenu en raison de l'effet de "l'immigrant en bonne santé ». De plus, comme riches et pauvres cohabitent au sein de nombreux quartiers, l'utilisation des moyennes de quartier entraîne le classement d'un grand nombre de personnes dans une mauvaise catégorie, ce qui réduit les estimations de l'effet. Des études réalisées au Canada et aux États-Unis ont permis d'établir que le gradient de mortalité selon le revenu est plus prononcé lorsqu'on analyse les données en fonction du revenu familial plutôt qu'à partir de diverses mesures du revenu du quartier ${ }^{3-6}$.

Rattachement des auteurs :

1. Bureau de santé publique de la région de Peel, Brampton (Ontario), Canada

2. École de santé publique Dalla Lana, Université de Toronto, Toronto (Ontario), Canada

3. Département de santé communautaire et d'épidémiologie, Université Queen's, Kingston (Ontario), Canada

4. Faculté des sciences infirmières, Université McMaster, Hamilton (Ontario), Canada

5. Division de l'analyse de la santé, Statistique Canada, Ottawa (Ontario), Canada

6. Département d'épidémiologie et de médecine sociale, Université d'Ottawa, Ottawa (Ontario), Canada

Correspondance : Russell Wilkins, Division de l'analyse de la santé, Statistique Canada, RHC-24A, 100, promenade du Pré Tunney, Ottawa (Ontario) K1A 0T6; tél. : 613-951-5305; téléc. : 613-951-4936; courriel : russell.wilkins@statcan.gc.ca 
Certaines études sur la mortalité chez les immigrants au Canada ont fait abstraction des différences de revenu ${ }^{7-11}$, tandis que d'autres ont uniquement rajusté les données en fonction du revenu ou du revenu du quartier (au lieu de présenter les résultats en fonction du revenu) ${ }^{12-15}$.

Notre étude visait, d'une part, à présenter les différences de mortalité toutes causes confondues entre les quintiles de revenu à partir de microdonnées sur le revenu personnel et le revenu familial tirées du recensement et, d'autre part, à examiner la façon dont ces différences varient entre immigrants et non-immigrants. En nous appuyant sur les données de l'Étude canadienne de suivi de la mortalité selon le recensement de 1991 à 2001, nous avons calculé, pour l'ensemble de la ville de Toronto et de la région de Peel, des tables de survie selon le sexe et le quintile de suffisance du revenu, pour tous les membres de la cohorte ainsi que pour les immigrants et les non-immigrants pris séparément. À ce jour, aucune donnée sur la mortalité toutes causes confondues selon le revenu personnel ou familial comparant les résultats des immigrants et des non-immigrants n'a été publiée au Canada. Nous espérions que les résultats de cette étude nous aideraient à mieux comprendre les observations de notre étude précédente sur le sujet.

\section{Méthodologie}

\section{Source des données}

L'Étude canadienne de suivi de la mortalité selon le recensement de 1991 à 2001 réalisée par Statistique Canada repose sur une base de données de cohorte couplée de façon probabiliste, dans laquelle un échantillon de $15 \%$ ( $\mathrm{n}=2735$ 152) de la population hors établissement âgée de 25 ans et plus ayant rempli le questionnaire long du recensement de 1991 (la cohorte) a été couplé aux données sur les décès de la Base canadienne de données sur la mortalité qui couvre une période de près de onze ans (du 4 juin 1991 au 31 décembre 2001). Le fichier de données couplées contient de l'information relative à diverses caractéristiques personnelles, à la situation socioéconomique, aux limitations d'activité ainsi qu'à la cause et à la date du décès. D’autres précisions concernant la construction et le contenu du fichier de données couplées ont déjà été présentées ${ }^{5}$.

Pour cette étude, des données ont été extraites des divisions du recensement de 1991 qui correspondent à la région actuelle de Peel (Mississauga, Brampton et Caledon) et à la ville fusionnée de Toronto (comprenant les anciennes villes de Toronto, North York, York, Etobicoke et Scarborough, ainsi que l'ancien bourg d'East York). Nous avons regroupé les deux régions de façon à obtenir un échantillon dont la taille permettrait d'élaborer des tables de survie pour les vingt sous-populations étudiées, chacune nécessitant des données sur les décès par tranches d'âge de 5 ans. Les deux régions comptent un pourcentage semblable d'immigrants et se situent dans la même zone de marché du travail (la région métropolitaine de recensement de Toronto).

\section{Définitions}

Quintile de suffisance du revenu. Pour établir les quintiles de suffisance du revenu (tranches d'un cinquième de la population), on a déterminé, dans une étude antérieure ${ }^{5}$, le revenu total de toutes provenances, avant impôt et après transfert, de chaque famille économique et personne seule faisant partie de la population hors établissement visée par le recensement. On a calculé ensuite le rapport entre le revenu total et le seuil de faible revenu de 1991, selon la taille de chaque famille et de chaque collectivité. On a ensuite classé la population en fonction de ce rapport, âges et sexes confondus, par région métropolitaine, agglomération et région rurale de recensement pour chacune des provinces et on a finalement divisé le tout en cinquièmes.

Immigrant. Dans cette analyse, le terme « immigrant » fait référence aux personnes qui ne sont pas citoyennes canadiennes de naissance. Il comprend toutes les personnes qui sont ou ont déjà été immigrants au Canada ou qui ont le statut de résident non permanent selon le recensement de 1991. Certains immigrants habitent au Canada depuis de nombreuses années, tandis que d'autres viennent tout juste d'arriver; presque tous sont nés à l'étranger.
Non-immigrant. Dans le cadre de la présente analyse, le terme «non-immigrant » fait référence aux citoyens canadiens de naissance. Presque tous les non-immigrants sont nés au Canada.

\section{Techniques d'analyse}

Analyse de la mortalité. Pour chaque membre de la cohorte, les jours-personnes de suivi ont été calculés pour la période allant du début de l'étude (le 4 juin 1991) à la date du décès, de l'émigration (connue pour 1991 seulement) ou de la fin de l'étude (le 31 décembre 2001). Ensuite, les années-personnes à risque ont été calculées en divisant les jours-personne de suivi par 365,25.

Les tables de survie abrégées de la période visée pour chaque sexe et chaque quintile, de même que les erreurs-types et les intervalles de confiance (IC) à $95 \%$ correspondants ont été calculés d'après la méthode de Chiang ${ }^{16}$. Ces calculs ont été effectués après la conversion de l'âge au début de l'étude à l'âge au début de chaque année de suivi, tandis que les décès et les années-personnes à risque ont été calculés séparément pour chaque année (ou année partielle) de suivi. Les décès et les années-personnes à risque ont par la suite été regroupés par tranches d'âge de cinq ans selon l'âge au début de chaque année de suivi, avant le calcul des tables de survie ${ }^{5}$.

\section{Résultats}

\section{Membres de la cohorte et décès au cours de la période de suivi}

La cohorte de départ comptait 287500 membres vivant soit dans la ville de Toronto (220 400 personnes), soit dans la région de Peel (67 100 personnes), dont $53 \%$ d'immigrants. Au cours de la période de suivi, 25648 membres admissibles de cette cohorte sont décédés (12 134 immigrants, 13514 non-immigrants).

Le tableau 1 présente le nombre de membres, les années-personnes à risque et le nombre de décès selon le quintile de revenu pour tous les membres de la cohorte, ainsi que pour les immigrants et les non-immigrants en fonction du sexe. 
Moins de $20 \%$ des membres de la cohorte se situaient dans le quintile de revenu inférieur et plus de $20 \%$ dans le quintile supérieur. Cette observation s'explique principalement par le fait que les données sur les membres de la cohorte devaient être couplées aux données sur les déclarations de revenus pour que l'on puisse effectuer le suivi de la mortalité, et les personnes ayant un revenu élevé sont davantage susceptibles de produire une déclaration de revenus.

\section{Caractéristiques socioéconomiques de chaque quintile de revenu}

Le tableau 2 présente pour tous les membres de la cohorte, immigrants et non-immigrants des deux sexes confondus, diverses

TABLEAU 1

Membres de la cohorte, années personnes à risque et décès au cours de la période étudiée, selon le quintile de suffisance du revenu, le sexe et le statut d'immigrant, ville de Toronto et région de Peel, 1991 à 2001 (personnes de 25 ans et plus ne vivant pas dans un établissement au début de l'étude)

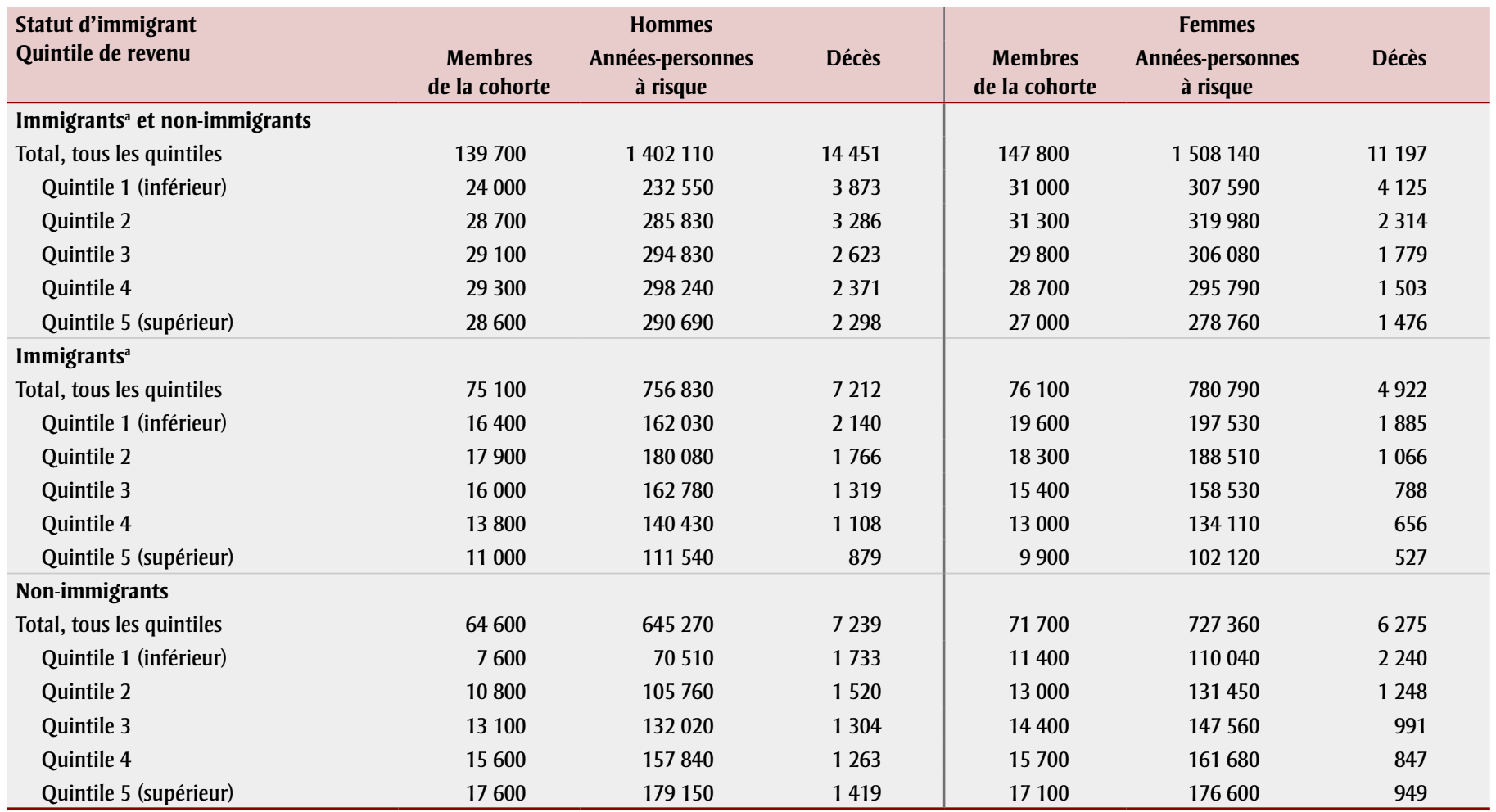

Source : Tableaux spéciaux tirés de l'Étude canadienne de suivi de la mortalité selon le recensement de 1991 à $2001^{5}$.

Remarque : Le nombre de membres de la cohorte et le nombre d'années personnes à risque ont été arrondis indépendamment (respectivement à la centaine et à la dizaine la plus proche).

${ }^{a}$ Nés à l'étranger, y compris les résidents non permanents.

TABLEAU 2

Caractéristiques des membres de la cohorte (immigrants et non-immigrants confondus) pour chaque quintile de suffisance du revenu, ville de Toronto et région de Peel, au début de l'étude, 1991

\begin{tabular}{|c|c|c|c|c|c|c|c|c|}
\hline \multirow[b]{2}{*}{ Quintile de revenu } & \multicolumn{8}{|c|}{ Pourcentage du nombre total de membres dans le quintile, \% } \\
\hline & Total & $\begin{array}{c}\text { Nés à } \\
\text { l'étranger }\end{array}$ & $\begin{array}{c}\text { Nouveaux } \\
\text { immigrants }^{b}\end{array}$ & $\begin{array}{l}\text { Minorités } \\
\text { visibles }^{\mathrm{C}}\end{array}$ & $\begin{array}{c}\text { Sans diplôme } \\
\text { d'études } \\
\text { secondaires }\end{array}$ & $\begin{array}{c}\text { Diplôme } \\
\text { universitaire }\end{array}$ & $\begin{array}{l}\text { Paiements de } \\
\text { transfert du } \\
\text { gouvernement }^{\mathrm{d}}\end{array}$ & Autochtones $^{\mathrm{e}}$ \\
\hline Total, tous les quintiles & 100,0 & 52,8 & 10,7 & 24,0 & 31,2 & 19,8 & 14,5 & 0,8 \\
\hline Quintile 1 (inférieur) & 100,0 & 65,7 & 23,0 & 35,2 & 46,4 & 10,4 & 41,0 & 1,0 \\
\hline Quintile 2 & 100,0 & 60,5 & 14,1 & 29,5 & 37,5 & 12,2 & 15,9 & 0,8 \\
\hline Quintile 3 & 100,0 & 53,5 & 8,7 & 24,0 & 31,1 & 16,1 & 8,7 & 0,8 \\
\hline Quintile 4 & 100,0 & 46,4 & 5,1 & 18,4 & 25,1 & 22,1 & 5,3 & 0,7 \\
\hline Quintile 5 (supérieur) & 100,0 & 37,9 & 2,8 & 12,8 & 16,0 & 38,8 & 2,4 & 0,6 \\
\hline
\end{tabular}

Source : Tableaux spéciaux tirés de l'Étude canadienne de suivi de la mortalité selon le recensement de 1991 à $2001^{5}$.

${ }^{a}$ Près de $100 \%$ des immigrants, mais moins de $1 \%$ des non immigrants, étaient nés à l'étranger.

b Personnes ayant immigré entre 1986 et 1991.

c Au Canada, le terme «minorité visible » ne comprend pas les peuples autochtones; $43 \%$ des immigrants et $3 \%$ des non immigrants appartenaient aux minorités visibles.

d Pourcentage des membres de la cohorte ayant pour principale source de revenu des paiements de transfert du gouvernement.

e Personnes d'ascendance autochtone ou Indiens inscrits; presque en totalité (96\%) des non-immigrants. 
caractéristiques socioéconomiques pour chaque quintile de suffisance du revenu au début de l'étude, caractéristiques exprimées sous forme de pourcentage du nombre total de membres dans chaque quintile. Puisque toutes les caractéristiques présentées variaient clairement suivant le revenu, nous n'avons relevé que les valeurs les plus élevées et les plus basses de chaque série.

Comparativement aux membres de la cohorte qui se situaient dans le quintile de revenu supérieur, ceux qui se trouvaient dans le quintile inférieur étaient beaucoup plus nombreux à ne pas avoir de diplôme d'études secondaires (46\% contre $16 \%$ ) et à avoir pour principale source de revenu des paiements de transfert du gouvernement (41\% contre $2 \%$ ). De plus, ils étaient moins nombreux à détenir un diplôme universitaire (10 \% contre $39 \%$ ).

Près de la totalité des immigrants, mais moins de $1 \%$ des non-immigrants, étaient nés à l'étranger. Dans le quintile de revenu supérieur, le pourcentage de membres nés à l'étranger s'établissait à $38 \%$, par rapport à $66 \%$ dans le quintile inférieur. Le pourcentage de nouveaux immigrants (entre 1986 et 1991) se situait à $3 \%$ dans le quintile de revenu supérieur, comparativement à $23 \%$ dans le quintile inférieur.
Au Canada, le terme « minorité visible » ne comprend pas les peuples autochtones ${ }^{5}$. Dans la cohorte Toronto-Peel, le pourcentage de membres d'une minorité visible s'établissait à $13 \%$ dans le quintile de revenu supérieur, par rapport à $35 \%$ dans le quintile inférieur. Presque tous les membres autochtones de la cohorte (96\%) étaient des non-immigrants, mais le pourcentage d'Autochtones était faible $(\leq 1 \%)$ dans tous les quintiles.

Le tableau 3 présente les pourcentages globaux liés à chacune des caractéristiques pour les immigrants et les non-immigrants pris séparément, ainsi que les pourcentages pour les immigrants et les non-immigrants selon le quintile de revenu. Dans l'ensemble, un cinquième $(20 \%)$ des immigrants étaient de nouveaux immigrants (entre 1986 et 1991), mais ils comptaient pour plus du tiers (35\%) du quintile de revenu inférieur et pour moins du dixième ( $8 \%$ ) $\mathrm{du}$ quintile supérieur. Sans surprise, un nombre beaucoup plus élevé d'immigrants que de non-immigrants faisait partie des minorités visibles ( $43 \%$ contre $3 \%$ ), bien que parmi les immigrants, le pourcentage de membres des minorités visibles variait, passant de plus de la moitié (52\%) dans le quintile de revenu inférieur à moins du tiers (30 \%) dans le quintile supérieur. Plus du tiers des immigrants n'avait pas obtenu de diplôme d'études secondaires (36 \%), comparativement au quart environ, chez les non-immigrants (26\%). Par rapport aux non-immigrants, les immigrants étaient moins nombreux à détenir un diplôme universitaire (16\% contre $24 \%$ ), mais un peu plus nombreux à avoir pour principale source de revenu des paiements de transfert du gouvernement (16\% contre $13 \%)$. Parmi les non-immigrants, le pourcentage d'Autochtones s'établissait à $3 \%$ dans le quintile de revenu inférieur et à moins de $1 \%$ dans le quintile supérieur.

\section{Disparités dans le nombre d'années de vie restantes à 25 ans}

L'espérance de vie restante à 25 ans (qui présuppose d'avoir survécu jusqu'à cet âge) est présentée dans le tableau 4. Pour tous les membres de la cohorte de la région étudiée (tant chez les hommes que chez les femmes et tant chez les immigrants que chez les non-immigrants), on observe un gradient manifeste, l'espérance de vie augmentant graduellement avec le quintile de revenu. La différence entre l'espérance de vie restante des membres du quintile de revenu inférieur et celle des membres du quintile supérieur atteignait 5,3 ans chez les hommes et 3,3 ans chez les femmes.

Chez les non-immigrants, le gradient d'espérance de vie restante selon le revenu était plus prononcé et affichait une nette progression en escalier entre les divers

TABLEAU 3

Caractéristiques des membres de la cohorte, immigrants et non immigrants pris séparément, pour chaque quintile de suffisance du revenu, ville de Toronto et région de Peel, au début de l'étude, 1991 (pourcentage du total de la rangée pour les immigrants et les non-immigrants)

\begin{tabular}{|c|c|c|c|c|c|c|c|c|c|c|c|c|}
\hline \multirow[b]{2}{*}{$\begin{array}{l}\text { Quintile } \\
\text { de revenu }\end{array}$} & \multicolumn{2}{|c|}{ Nouveaux immigrants ${ }^{\mathrm{a}}$} & \multicolumn{2}{|c|}{ Minorités visibles $^{\mathbf{b}}$} & \multicolumn{2}{|c|}{$\begin{array}{c}\text { Sans diplôme } \\
\text { d'études secondaires }\end{array}$} & \multicolumn{2}{|c|}{ Diplôme universitaire } & \multicolumn{2}{|c|}{$\begin{array}{c}\text { Paiements de } \\
\text { transfert du } \\
\text { gouvernement }\end{array}$} & \multicolumn{2}{|c|}{ Autochtones $^{d}$} \\
\hline & Immigrant & $\begin{array}{c}\text { Non- } \\
\text { immigrant }\end{array}$ & Immigrant & $\begin{array}{c}\text { Non- } \\
\text { immigrant }\end{array}$ & Immigrant & $\begin{array}{c}\text { Non- } \\
\text { immigrant }\end{array}$ & Immigrant & $\begin{array}{c}\text { Non- } \\
\text { immigrant }\end{array}$ & Immigrant & $\begin{array}{c}\text { Non- } \\
\text { immigrant }\end{array}$ & Immigrant & $\begin{array}{c}\text { Non- } \\
\text { immigrant }\end{array}$ \\
\hline $\begin{array}{l}\text { Total, tous } \\
\text { les quintiles }\end{array}$ & 20,3 & 0,0 & 43,1 & 2,8 & 36,4 & 25,5 & 16,3 & 23,7 & 16,1 & 12,6 & 0,1 & 1,6 \\
\hline $\begin{array}{l}\text { Quintile } 1 \\
\text { (inférieur) }\end{array}$ & 35,2 & 0,0 & 52,1 & 3,3 & 45,7 & 47,7 & 10,8 & 9,8 & 36,6 & 49,2 & 0,0 & 2,7 \\
\hline Quintile 3 & 16,4 & 0,0 & 42,7 & 2,6 & 35,7 & 25,8 & 14,5 & 17,9 & 9,7 & 7,6 & 0,1 & 1,6 \\
\hline Quintile 4 & 11,0 & 0,0 & 36,5 & 2,8 & 30,7 & 20,2 & 19,2 & 24,6 & 6,8 & 3,9 & 0,1 & 1,3 \\
\hline $\begin{array}{l}\text { Quintile 5 } \\
\text { (supérieur) }\end{array}$ & 7,6 & 0,0 & 29,7 & 2,6 & 21,6 & 12,6 & 33,0 & 42,4 & 3,5 & 1,7 & 0,1 & 0,9 \\
\hline
\end{tabular}

Source : tableaux spéciaux tirés de l'Étude canadienne de suivi de la mortalité selon le recensement de 1991 à $2001^{5}$.

a Personnes ayant immigré entre 1986 et 1991.

${ }^{\mathrm{b}}$ Au Canada, le terme « minorité visible » ne comprend pas les peuples autochtones; $43 \%$ des immigrants et $3 \%$ des non immigrants appartenaient aux minorités visibles.

c Pourcentage des membres de la cohorte ayant pour principale source de revenu des paiements de transfert du gouvernement.

d Personnes d'ascendance autochtone ou Indiens inscrits; presque en totalité (96\%) des non-immigrants. 
TABLEAU 4

Espérance de vie restante à 25 ans (qui présuppose d'avoir survécu jusqu'à cet âge) selon le quintile de suffisance du revenu, le sexe et le statut d'immigrant, ville de Toronto et région de Peel, 1991 à 2001

\begin{tabular}{|c|c|c|}
\hline $\begin{array}{l}\text { Statut d'immigrant } \\
\text { Quintile de revenu }\end{array}$ & $\begin{array}{c}\text { Hommes } \\
\text { Années (IC à } 95 \% \text { ) }\end{array}$ & $\begin{array}{c}\text { Femmes } \\
\text { Années (IC à } 95 \%)\end{array}$ \\
\hline \multicolumn{3}{|c|}{ Immigrants ${ }^{\mathrm{a}}$ et non-immigrants } \\
\hline Total, tous les quintiles & $53,7 \quad(53,5$ à 53,9$)$ & $59,3 \quad(59,1$ à 59,5$)$ \\
\hline Quintile 1 (inférieur) & $50,6 \quad(50,2$ à 51,0$)$ & $57,1 \quad(56,7$ à 57,5$)$ \\
\hline Quintile 2 & $53,0 \quad(52,6$ à 53,4) & $59,2 \quad(58,8$ à 59,6$)$ \\
\hline Quintile 3 & $54,0 \quad(53,6$ à 54,4$)$ & $59,7 \quad(59,2$ à 60,1$)$ \\
\hline Quintile 4 & $55,0 \quad(54,6$ à 55,4$)$ & $60,6 \quad(60,2$ à 61,1$)$ \\
\hline Quintile (supérieur) & $56,0 \quad(55,5$ à 56,4$)$ & $60,4 \quad(60,0$ à 60,9$)$ \\
\hline Différence Q5-Q1 & $5,3 \quad(4,7$ à 5,9) & $3,3 \quad(2,7$ à 3,9$)$ \\
\hline \multicolumn{3}{|l|}{ Immigrants $^{\mathrm{a}}$} \\
\hline Total, tous les quintiles & $55,4 \quad(55,1$ à 55,6$)$ & $60,9 \quad(60,6$ à 61,1$)$ \\
\hline Quintile 1 (inférieur) & $53,8 \quad(53,3$ à 54,3$)$ & $60,3 \quad(59,8$ à 60,8$)$ \\
\hline Quintile 2 & $55,0 \quad(54,5$ à 55,5$)$ & $60,7 \quad(60,1$ à 61,2$)$ \\
\hline Quintile 3 & $55,6 \quad(55,0$ à 56,1$)$ & $60,9 \quad(60,3$ à 61,5$)$ \\
\hline Quintile 4 & $56,4 \quad(55,7$ à 57,0$)$ & $61,2 \quad(60,5$ à 61,9) \\
\hline Quintile (supérieur) & $57,6 \quad(56,9$ à 58,3$)$ & $61,1 \quad(60,3$ à 61,8$)$ \\
\hline Différence Q5-Q1 & $3,8 \quad(3,0$ à 4,7) & $0,8 \quad(-0,1$ à 1,7$)$ \\
\hline \multicolumn{3}{|l|}{ Non-immigrants } \\
\hline Total, tous les quintiles & $51,7 \quad(51,4$ à 52,0$)$ & $57,6 \quad(57,4$ à 57,9$)$ \\
\hline Quintile 1 (inférieur) & $45,1 \quad(44,4$ à 45,9$)$ & $52,5 \quad(51,8$ à 53,2$)$ \\
\hline Quintile 2 & $49,7 \quad(49,0$ à 50,4$)$ & $57,3 \quad(56,7$ à 58,0$)$ \\
\hline Quintile 3 & $51,7 \quad(51,1$ à 52,3$)$ & $58,4 \quad(57,7$ à 59,0$)$ \\
\hline Quintile 4 & $53,6 \quad(53,0$ à 54,1$)$ & $60,1 \quad(59,4$ à 60,7$)$ \\
\hline Quintile (supérieur) & $55,0 \quad(54,5$ à 55,5$)$ & $60,0 \quad(59,4$ à 60,5$)$ \\
\hline Différence Q5-Q1 & $9,8 \quad(8,9$ à 10,7$)$ & $7,4 \quad(6,5$ à 8,3$)$ \\
\hline
\end{tabular}

Source : Tableaux spéciaux tirés de l'Étude canadienne de suivi de la mortalité selon le recensement de 1991 à $2001^{5}$.

Abréviations : IC, intervalle de confiance; $Q$, quintile.

${ }^{a}$ Nés à l'étranger, y compris les résidents non permanents.

quintiles de revenu, tant chez les hommes que chez les femmes. L'écart entre les quintiles de revenu inférieur et supérieur se situait à 9,8 ans chez les hommes et à 7,4 ans chez les femmes.

Chez les immigrants, le gradient d'espérance de vie restante selon le revenu était beaucoup moins prononcé. L'écart entre les quintiles de revenu supérieur et inférieur s'établissait à 3,8 ans chez les hommes et à 0,8 an chez les femmes.

Soulignons que dans chaque quintile de revenu et pour chacun des sexes, l'espérance de vie restante des immigrants dépassait toujours celle des non-immigrants. Toutefois, les différences entre les immigrants et les non-immigrants étaient moins marquées dans le quintile supérieur (2,6 ans chez les hommes et 1,1 an chez les femmes) et plus marquées dans le quintile inférieur (8,7 ans chez les hommes et 7,8 ans chez les femmes).

\section{Disparités dans la probabilité} de survie jusqu'à l'âge de 75 ans

La probabilité de survie jusqu'à l'âge de 75 ans (à la condition d'avoir survécu jusqu'à l'âge de 25 ans) est présentée dans le tableau 5. Comme dans le cas de l'espérance de vie à 25 ans, le pourcentage d'hommes et de femmes pouvant espérer vivre jusqu'à l'âge de 75 ans augmentait graduellement avec le quintile de revenu pour tous les membres de la cohorte, tant chez les immigrants que chez les non-immigrants. Cependant, les disparités étaient beaucoup plus marquées chez les non-immigrants.

Pour tous les membres de la cohorte, tant chez les hommes que chez les femmes, on observe un gradient manifeste, la probabilité de survie jusqu'à l'âge de 75 ans augmentant graduellement avec le quintile de revenu. Chez les hommes, la probabilité de survie jusqu'à l'âge de 75 ans s'établissait à $57 \%$ dans le quintile de revenu inférieur et à $74 \%$ dans le quintile supérieur (une différence de 17 points de pourcentage). Chez les femmes, la probabilité de survie jusqu'à l'âge de 75 ans se situait à $73 \%$ dans le quintile de revenu inférieur et à $84 \%$ dans le quintile supérieur (une différence de 10 points de pourcentage).

Chez les hommes non immigrants, la probabilité de survie jusqu'à l'âge de 75 ans atteignait $40 \%$ dans le quintile de revenu inférieur et $73 \%$ dans le quintile supérieur (une différence de 33 points de pourcentage). Chez les hommes immigrants, la probabilité de survie jusqu'à l'âge de 75 ans s'établissait à $67 \%$ dans le quintile de revenu inférieur et à $76 \%$ dans le quintile supérieur (une différence de 9 points de pourcentage).

Chez les femmes non immigrantes, la probabilité de survie jusqu'à l'âge de 75 ans se situait à $61 \%$ dans le quintile de revenu inférieur et à $82 \%$ dans le quintile supérieur (une différence de 21 points de pourcentage). Chez les femmes immigrantes, la probabilité de survie jusqu'à l'âge de 75 ans atteignait $81 \%$ dans le quintile de revenu inférieur et $85 \%$ dans le quintile supérieur (une différence de 4 points de pourcentage).

Soulignons que dans chaque quintile de revenu et pour chacun des sexes, la probabilité de survie jusqu'à l'âge de 75 ans des immigrants dépassait toujours celle des non-immigrants. Néanmoins, l'écart le plus grand entre les immigrants et les nonimmigrants était observé dans le quintile de revenu inférieur (28 points de pourcentage chez les hommes et 20 points de pourcentage chez les femmes) et l'écart le plus faible, dans le quintile supérieur (3 points de pourcentage, tant chez les hommes que chez les femmes).

\section{Analyse}

Les résultats de la présente étude montrent clairement que l'espérance de vie restante à 25 ans et la probabilité de survie jusqu'à l'âge de 75 ans des hommes et des femmes de la ville de Toronto et de la région de Peel diffèrent considérablement selon le quintile 
TABLEAU 5

Probabilité de survie jusqu'à l'âge de 75 ans (à la condition d'avoir survécu jusqu'à l'âge de 25 ans) selon le quintile de suffisance du revenu, le sexe et le statut d'immigrant, ville de Toronto et région de Peel, 1991 à 2001

\begin{tabular}{|c|c|c|c|c|}
\hline \multirow{2}{*}{$\begin{array}{l}\text { Statut d'immigrant } \\
\text { Quintile de revenu }\end{array}$} & \multicolumn{4}{|c|}{ Probabilité (\%) (IC à $95 \%$ ) } \\
\hline & \multicolumn{2}{|r|}{ Hommes } & \multicolumn{2}{|r|}{ Femmes } \\
\hline \multicolumn{5}{|c|}{ Immigrants et non-immigrants } \\
\hline Total, tous les quintiles & 66,9 & (66,3 à 67,5) & 80,1 & (79,5 à 80,6) \\
\hline Quintile 1 (inférieur) & 57,0 & (55,5 à 58,5) & 73,3 & $(72,0$ à 74,5$)$ \\
\hline Quintile 2 & 64,1 & (62,7 à 65,5) & 79,5 & (78,4 à 80,7) \\
\hline Quintile 3 & 67,3 & $(66,0$ à 68,7) & 81,8 & $(80,6$ à 82,9) \\
\hline Quintile 4 & 69,5 & $(68,2$ à 70,8) & 82,9 & $(81,7$ à 84,1$)$ \\
\hline Quintile (supérieur) & 74,1 & (72,9 à 75,4) & 83,6 & $(82,4$ à 84,7$)$ \\
\hline Différence Q5-Q1 & 17,2 & $(15,2$ à 19,1$)$ & 10,3 & (8,6 à 12,0) \\
\hline \multicolumn{5}{|l|}{ Immigrants ${ }^{\mathrm{a}}$} \\
\hline Total, tous les quintiles & 71,5 & (70,7 à 72,3) & 83,6 & (82,9 à 84,3) \\
\hline Quintile 1 (inférieur) & 67,2 & $(65,4$ à 69,1) & 81,0 & (79,5 à 82,4) \\
\hline Quintile 2 & 69,3 & $(67,6$ à 71,0) & 82,4 & (80,9 à 83,8) \\
\hline Quintile 3 & 72,3 & (70,6 à 74,0) & 85,1 & $(83,7$ à 86,6$)$ \\
\hline Quintile 4 & 73,1 & (71,3 à 74,9) & 85,3 & $(83,7$ à 86,9$)$ \\
\hline Quintile (supérieur) & 75,8 & (74,0 à 77,7) & 85,4 & $(83,6$ à 87,2$)$ \\
\hline Différence Q5-Q1 & 8,6 & (6,0 à 11,2) & 4,4 & (2,1 à 6,7) \\
\hline \multicolumn{5}{|l|}{ Non-immigrants } \\
\hline Total, tous les quintiles & 61,0 & $(60,0$ à 62,0) & 76,0 & $(75,2$ à 76,9$)$ \\
\hline Quintile 1 (inférieur) & 39,7 & (37,3 à 42,2) & 60,9 & (58,6 à 63,1) \\
\hline Quintile 2 & 54,5 & (52,0 à 56,9) & 75,1 & (73,2 à 77,1) \\
\hline Quintile 3 & 59,7 & (57,5 à 61,9) & 77,6 & (75,8 à 79,5) \\
\hline Quintile 4 & 65,1 & (63,1 à 67,2) & 80,2 & (78,5 à 82,0) \\
\hline Quintile (supérieur) & 72,9 & (71,2 à 74,6) & 82,3 & $(80,8$ à 83,9$)$ \\
\hline Différence Q5-Q1 & 33,1 & (30,2 à 36,1) & 21,5 & $(18,7$ à 24,2$)$ \\
\hline
\end{tabular}

Source : Tableaux spéciaux tirés de l’Étude canadienne de suivi de la mortalité selon le recensement de 1991 à $2001^{5}$.

Abréviations : IC, intervalle de confiance; $Q$, quintile.

${ }^{a}$ Nés à l'étranger, y compris les résidents non permanents.

de revenu. Cela n'a rien d'étonnant, mais il nous fallait des données sur le revenu personnel et le revenu familial pour le démontrer. Nous avons également constaté que les disparités dans la mortalité selon le revenu étaient beaucoup plus prononcées lorsque l'analyse se limitait aux nonimmigrants. De plus, dans chacun des quintiles de revenu, les immigrants obtenaient des résultats plus favorables que les non-immigrants, mais l'avantage des immigrants était particulièrement marqué dans les quintiles de revenu inférieurs. On n’avait encore jamais démontré, au Canada, la plus grande disparité de mortalité selon le revenu chez les non-immigrants (par rapport aux immigrants), ni l'avantage supérieur des immigrants des groupes de revenu inférieurs en matière de survie.

Notre précédente étude, de faible étendue géographique, ne nous avait pas permis de détecter les écarts dans l'espérance de vie selon le quintile de revenu du quartier. Cela s'explique probablement par la confusion considérable causée par la présence d'un pourcentage beaucoup plus élevé que la moyenne d'immigrants, dont le taux de mortalité est faible (et notamment de nouveaux immigrants, dont le taux de mortalité est particulièrement faible) au sein de quartiers, par ailleurs à faible revenu, présentant un taux de mortalité élevé. Cela pourrait également s'expliquer par une classification non différentielle découlant de l'utilisation des moyennes de secteurs de recensement plutôt que du revenu personnel et du revenu familial.

En ce qui concerne l'espérance de vie restante et la probabilité de survie jusqu'à l'âge de 75 ans, nos résultats viennent non seulement confirmer les études précédentes en faisant ressortir des disparités importantes selon le revenu, dans l'ensemble, mais ils révèlent également que le gradient de mortalité selon le revenu est beaucoup plus prononcé chez les non-immigrants que chez les immigrants.

L'effet de l'immigrant en bonne santé reflète vraisemblablement un degré élevé d'autosélection. Premièrement, les personnes en mauvaise santé sont moins susceptibles d'essayer d'immigrer. Deuxièmement, le processus d'immigration exige des immigrants qu'ils se soumettent à un examen médical pour pouvoir être admis au Canada et les immigrants sont choisis en fonction de leur richesse, de leur employabilité ${ }^{9}$, de leur niveau de scolarité et de leurs aptitudes linguistiques ${ }^{17}$. Troisièmement, les comportements malsains tels que le tabagisme, la consommation excessive d'alcool et une mauvaise alimentation sont généralement moins fréquents chez les immigrants que chez les non-immigrants ${ }^{9,18}$.

Des études ont révélé qu'en règle générale, les immigrants sont en meilleure santé que les non-immigrants. Ce phénomène a été observé pour toute une gamme de maladies chroniques, de même que pour les incapacités, la dépendance, l'espérance de vie et l'espérance de vie sans incapacité ${ }^{17-24}$. Bien que les immigrants soient habituellement en excellente santé à leur arrivée au Canada, leur état de santé tend, au fil du temps, à ressembler davantage à celui de la population née au Canada ${ }^{7,25}$. Des études ont notamment permis d'établir que les nouveaux immigrants présentent des taux de mortalité plus faibles que les immigrants de plus longue date ${ }^{5}$. L'écart plus important entre les immigrants et les non-immigrants que nous avons observé dans les quintiles de revenu inférieurs (par rapport aux quintiles supérieurs) semble être associé, du moins en partie, au pourcentage plus élevé de nouveaux immigrants dans les quintiles de revenu inférieurs. Les prochaines études de puissance suffisante devraient pouvoir tenir compte de ce facteur de confusion.

\section{Limites}

L'Étude canadienne de suivi de la mortalité selon le recensement de 1991 à 2001 a exclu les personnes non recensées lors du recensement de 1991 (environ 3,4 \% de la population totale). Les personnes non recensées étaient plus susceptibles d'être jeunes, mobiles, à faible revenu, 
d'ascendance autochtone ${ }^{26}$ ou sans abri. De plus, les personnes résidant dans un établissement de soins de longue durée, une résidence pour personnes âgées ou une prison (qui n’ont pas été dénombrées par le questionnaire long) et les personnes n'ayant pas produit de déclaration de revenus pour les années d'imposition 1990 et 1991 (cette information étant nécessaire pour le couplage des données) ont été exclues de la cohorte. Par conséquent, l'espérance de vie restante de la cohorte, à l'échelle du Canada, atteignait une année de plus chez les hommes et deux années de plus chez les femmes que celle figurant dans les tables de survie de l'ensemble de la population canadienne ${ }^{5}$.

L'information sur le revenu familial et sur le lieu de résidence n'était disponible qu'au début de l'étude. Puisqu'on peut s'attendre à ce que ces éléments changent au fil du temps, il aurait été préférable de connaître le revenu et le lieu de résidence pour chacune des années de suivi.

Comme cette analyse se limitait à la ville de Toronto et à la région de Peel, notre cohorte était trop petite pour nous permettre d'établir, au delà des caractéristiques de population, davantage de distinctions entre les nouveaux immigrants et les immigrants de longue date. De futures analyses sur l'ensemble de la cohorte devraient s'y consacrer, en plus d'examiner d'autres facteurs tels que le pays ou la région d'origine et l'appartenance à une minorité visible, afin de mieux expliquer les tendances selon le revenu observées dans ces données ${ }^{27}$.

\section{Conclusion}

Nos résultats soulignent l'importance d'utiliser les données individuelles et familiales pour l'analyse des disparités en matière de santé selon le revenu au sein d'une population aussi diversifiée que celle de la plus grande région métropolitaine au Canada. Ils font également ressortir l'importance de prendre en compte le statut d'immigrant et le caractère récent de l'immigration afin d'expliquer la relation entre le revenu et deux indicateurs de la santé fondamentaux, soit l'espérance de vie restante à 25 ans et la probabilité de survie jusqu'à l'âge de 75 ans. Cet aspect est particulièrement important dans des endroits comme la ville de Toronto et la région de Peel, qui comptent une proportion très élevée d'immigrants, dont de nombreux nouveaux immigrants.

Bien que les registres de l'état civil (décès et naissances) recueillent des données sur le lieu de naissance du défunt ou de la mère, cette information est souvent écartée au moment de la compilation et de l'analyse des données. Dans d'autres ensembles de données administratives comme les registres du cancer et les données sur la morbidité hospitalière, aucune information sur le lieu de naissance n'est recueillie. La présente étude montre l'importance de recueillir et d'analyser ce type de données, non seulement pour mieux comprendre la relation entre l'immigration et la santé (ce qui demeure important), mais aussi pour préciser l'étendue et la nature des disparités en matière de santé, de façon plus générale, en fonction de la situation socioéconomique.

Des travaux ultérieurs pourraient envisager les moyens d'inclure la morbidité et la mortalité des pensionnaires d'établissements institutionnels (le segment de la population qui présente le plus d'incapacités) et des personnes de moins de 25 ans afin d'obtenir un portrait plus global de la morbidité et de la mortalité selon le statut socioéconomique ${ }^{3}$. Par ailleurs, l'analyse des causes de décès dans chaque quintile nous permettrait d'en apprendre davantage sur les efforts de prévention qui pourraient contribuer à réduire les disparités en matière de santé liées aux conditions socioéconomiques.

\section{Remerciements}

Une partie importante du financement consacré à la réalisation de l'Étude canadienne de suivi de la mortalité selon le recensement de 1991 à 2001, sur laquelle se fondent nos travaux, a été fournie par l'Initiative sur la santé de la population canadienne de l'Institut canadien d'information sur la santé.

Nous tenons à remercier les registraires de l'état civil des provinces et des territoires du Canada pour les données sur la mortalité, Statistique Canada, qui a mené le recensement de 1991, ainsi que tous les Canadiens, dont les réponses fournies au questionnaire long du recensement ont constitué le fondement des présentes analyses.

\section{Annexe}

TABLEAU A

Espérance de vie restante à 25 ans selon le quintile de revenu du quartier, région de Peel, 2005

\begin{tabular}{|c|c|c|}
\hline \multirow[t]{2}{*}{ Quintile de revenu du quartier } & \multicolumn{2}{|c|}{ Années de vie restantes } \\
\hline & Hommes & Femmes \\
\hline Quintile 1 (inférieur) & 56,6 & 61,1 \\
\hline Quintile 2 & 57,3 & 60,6 \\
\hline Quintile 3 & 56,8 & 61,0 \\
\hline Quintile 4 & 57,3 & 59,9 \\
\hline Quintile 5 (supérieur) & 57,0 & 59,4 \\
\hline Différence : Q5-Q1 & $+0,4$ & $-1,7$ \\
\hline
\end{tabular}

Source : Stratton J, Mowat DL, Wong KP. Remaining life expectancy at age 25, by neighbourhood income quintile, Peel Region, 2005. Brampton, Ontario: Region of Peel Public Health, 2010. Tableaux spéciaux tirés de la Base de données sur la mortalité de l’Ontario (2005), du SIPSS (Système informatique de planification des services de santé), du ministère de la Promotion de la santé, et des profils de secteurs de recensement, 2006, Statistique Canada.

Remarque : Les quintiles de revenu du quartier reposent sur le pourcentage de personnes, par secteur de recensement, dont le revenu de la famille économique ou le revenu personnel était inférieur au seuil de faible revenu établi par Statistique Canada en fonction de la taille de la famille et de la taille de la collectivité. 


\section{Références}

1. Wolfson MC, Kaplan G, Lynch J, Ross N, Backlund E. Relation between income inequality and mortality: empirical demonstration. West $\mathrm{J}$ Med. 2000;172(1):22-4.

2. Wilkins R, Berthelot JM, Ng E. Tendances de la mortalité selon le revenu du quartier dans les régions urbaines du Canada, 1971-1996. Rapports sur la santé. 2002;13(suppl.):1-29.

3. McIntosh CN, Finès $\mathrm{P}$, Wilkins $\mathrm{R}$, Wolfson MC. Disparités selon le revenu dans l'espérance de vie ajustée sur la santé chez les adultes au Canada, 1991 à 2001. Rapports sur la santé. 2009;20(4):59-70.

4. Pampalon R, Hamel D, Gamache P. Une comparaison de données socio-économiques individuelles et géographiques pour la surveillance des inégalités sociales de santé au Canada. Rapports sur la santé. 2009;20(4):1-11.

5. Wilkins R, Tjepkema M, Mustard C, Choinière R. Étude canadienne de suivi de la mortalité selon le recensement, 1991 à 2001. Rapports sur la santé. 2008;19(3):27-48.

6. Lynch J, Harper S, Kaplan GA, Davey Smith G. Associations between income inequality and mortality among US states: the importance of time period and source of income data. Am J Public Health. 2005;95(8):1424-30.

7. Kliewer EV, Ward RH. Convergence of immigrant suicide rates to those in the destination country. Am J Epidemiol. 1988;127(3):640-53.

8. Sharma RD, Michalowski M, Verma RB. Mortality differentials among immigrant populations in Canada. Int Migr. 1990;28(4):443-50.

9. Chen J, Wilkins R, Ng E. Espérance de santé selon le statut d'immigrant, 1986 et 1991. Rapports sur la santé. 1996;8(3):31-41.

10. DesMeules M, Gold J, McDermott S, Cao Z, Payne J, Lafrance B, et al. Disparities in mortality patterns among Canadian immigrants and refugees, 1980-1998: results of a national cohort study. J Immigr Health. 2005;7(4):221-32.
11. Trovato F, Odynak D. Sex differences in life expectancy in Canada: immigrant and native-born populations. J Biosoc Sci. 2011;43(3):353-67.

12. Saposnik G, Redelmeier DA, Lu H, Fuller-Thomson E, Lonn E, Ray JG. Myocardial infarction associated with recency of immigration to Ontario. QJM. 2010;103(4):253-8

13. Pampalon R, Hamel D, Gamache P. Health inequalities, deprivation, immigration and aboriginality in Canada: a geographic perspective. Can J Public Health. 2010;101(6):470-4.

14. Ng E; Équipe de recherche de l'Initiative sur les données longitudinales administratives et sur la santé. Le point sur l'effet de l'immigrant en santé : mortalité selon la période d'immigration et le lieu de naissance. Ottawa (Ont.) : Statistique Canada, septembre 2011. 16 p. (Série de documents de travail de la recherche sur la santé) (Statistique Canada, $\mathrm{n}^{0}$ 82-622-X au catalogue $-n^{\circ} 008$ )

15. Ng E. Effet de l'immigrant en santé et taux de mortalité. Rapports sur la santé. 2011;22(4):1-6

16. Chiang CL. The life table and its applications. Éd. originale. Malabar (FL): Robert E Krieger Publ Co; 1984

17. Hyman I. Immigration and health: reviewing evidence of the healthy immigrant effect in Canada. CERIS Working Paper No. 55 Toronto (Ont.): Joint Centre of Excellence for Research on Immigration and Settlement; 2007.

18. Pérez CE. État de santé et comportement influant sur la santé des immigrants. Rapports sur la santé. 2002;13(suppl.):1-13.

19. Singh GK, Miller BA. Health, life expectancy, and mortality patterns among immigrant populations in the United States. Can J Public Health. 2004;95(3): I14-I21.

20. DesMeules M, Gold J, Kazanjian A, Manuel D, Payne J, Vissandée B, McDermott S, Mao Y. New approaches to immigrant health assessment. Can J Public Health. 2004;95(3):22-6
21. Singh GK, Siahpush M. All-cause and cause-specific mortality of immigrants and native born in the United States. Am J Public Health. 2001;91(3):392-9.

22. Singh GK, Hiatt RA. Trends and disparities in socioeconomic and behavioural characteristics, life expectancy, and cause-specific mortality of native-born and foreign-born populations in the United States, 1979-2003. Int J Epidemiol. 2006;35(4):903-19.

23. Hajat A, Blakely $T$, Dayal S, Jatrana S. Do New Zealand's immigrants have a mortality advantage? Evidence from the New Zealand Census-Mortality Study. Ethn Health. 2010;15(5):531-47.

24. Singh GK, Siahpush M. Ethnic-immigrant differentials in health behaviors, morbidity, and cause-specific mortality in the United States: an analysis of two national data bases. Hum Biol. 2002;74(1):83-109.

25. Newbold KB. Self-rated health within the Canadian immigrant population: risk and the healthy immigrant effect. Soc Sci Med. 2005;60(6):1359-70.

26. Statistique Canada. Couverture. Rapports techniques du recensement de 1991, Série des produits de référence. Ottawa (Ont.) : ministre de l'Industrie, des Sciences et de la Technologie; 1994 (Statistique Canada, $\mathrm{n}^{\circ}$ 92-341F au catalogue).

27. McDonald JT, Kennedy S. Insights into the 'healthy immigrant effect': health status and health service use of immigrants to Canada. Soc Sci Med. 2004;59(8):1613-27. 